\title{
Probing AGN triggering mechanisms through the starburstiness of the host galaxies
}

\author{
A. Lamastra, N. Menci, F. Fiore, P. Santini, A. Bongiorno, and E. Piconcelli
}

\begin{abstract}
INAF - Osservatorio Astronomico di Roma, via di Frascati 33, 00040 Monte Porzio Catone, Italy
\end{abstract} e-mail: alessandra.lamastra@oa-roma.inaf.it

Received 13 September 2013 / Accepted 15 October 2013

\begin{abstract}
We estimate the fraction of AGNs hosted in starburst galaxies $\left(f_{\text {bursty }}\right)$ as a function of the AGN luminosity predicted under the assumption that starburst events and AGN activity are triggered by galaxy interactions during their merging histories. The latter are described through Monte Carlo realizations, and are connected to star formation and BH accretion using a semi-analytic model of galaxy formation in a cosmological framework. The predicted fraction $f_{\text {bursty }}$ increases steeply with AGN luminosity from $\lesssim 0.2$ at $L_{\mathrm{X}} \lesssim 10^{44} \mathrm{erg} / \mathrm{s}$ to $\gtrsim 0.9$ at $L_{\mathrm{X}} \gtrsim 10^{45} \mathrm{erg} / \mathrm{s}$ over a wide redshift interval from $z \simeq 0$ to $z \simeq 6$. We compare the model predictions with new measurements of $f_{\text {bursty }}$ from a sample of X-ray selected AGNs in the XMM-COSMOS field at $0.3<z<2$, and from a sample of QSOs $\left(L_{\mathrm{X}} \gtrsim 10^{45} \mathrm{erg} / \mathrm{s}\right)$ in the redshift range $2<z<6.5$. We find preliminary indications that under conservative assumptions half of the QSO hosts are starburst galaxies. This result provides motivation for future systematic studies of the stellar properties of high luminosity AGN hosts in order to constrain AGN triggering mechanisms.
\end{abstract}

Key words. galaxies: active - galaxies: evolution - galaxies: fundamental parameters - galaxies: interactions - galaxies: starburst

\section{Introduction}

The tight correlations between the black hole (BH) mass and the properties of the host galaxy spheroid, including mass, luminosity, and stellar velocity dispersion (Kormendy \& Richstone 1995; Magorrian et al. 1998; Ho 1999; Gebhardt et al. 2000; Ferrarese \& Merritt 2000; Marconi \& Hunt 2003; Häring \& Rix 2004; Kormendy \& Bender 2009), imply a tight link between galaxy evolution and $\mathrm{BH}$ growth. Since the growth of BHs is mostly due to accretion of matter during active galactic nucleus (AGN) phases (Soltan 1982), these findings suggest that the mechanisms responsible for building up the stellar mass in spheroids are also related to the triggering of AGN activity. Theoretical models connecting AGN activity to the merging histories of the host galaxies naturally reproduce the observed BH mass-stellar mass relation in the local (e.g. Peng 2007; Bower et al. 2006; Croton 2006; Somerville et al. 2008; Marulli et al. 2008; Hirschmann et al. 2010) and higher redshift Universe (e.g. Lamastra et al. 2010). However, since the mass is an integrated quantity, these correlations could also originate from the hierarchical aggregation of mass (Jahnke \& Macciò 2011).

Further hints for the nature of AGN triggering mechanisms could be provided by the study of the correlation between the derivative of the $\mathrm{BH}$ mass and the stellar mass, namely, the accretion rate and the star formation rate (SFR). Most of the studies of this correlation have been done through observations in the characteristic emission bands of the two processes, namely, the far-infrared (FIR) emission from cold dust heated by the UV radiation of massive young stars and the hard X-ray emission from the hot corona of the AGN. Observational studies based on those indicators revealed a complex situation. In the local Universe Netzer (2009) found a strong correlation between the luminosity at $60 \mu \mathrm{m}$ and the AGN luminosity for optically-selected
AGNs over more than five orders of magnitude in luminosity. According to Rosario et al. (2012) the luminosity at $60 \mu \mathrm{m}$ is correlated to the AGN luminosity for AGNs with X-ray luminosities $L_{\mathrm{X}} \approx 10^{43} \mathrm{erg} / \mathrm{s}$ at $z<1$, while no correlation is found for AGNs of the same luminosity at higher redshift and for lower luminosity AGNs (see also Lutz et al. 2010; Shao et al. 2010). Mullaney et al. (2012b) found no evidence of any correlation between the X-ray and infrared luminosities of AGN with $L_{\mathrm{X}}=10^{42}-10^{44} \mathrm{erg} / \mathrm{s}$ up to $z=3$. However, the correlation arises at $z=1-2$ when stacked X-ray emission of undetected sources is taken into account (Mullaney et al. 2012a). Finally, Rovilos et al. (2012) found evidence for a correlation between $L_{\mathrm{X}}$ and the SFR per unit stellar mass of the host galaxy $\left(S S F R=S F R / M_{*}\right)$ for AGNs with $L_{\mathrm{X}}>10^{43} \mathrm{erg} / \mathrm{s}$ at $z>1$, and they did not find evidence for such correlation for lower luminosity systems or those at lower redshifts.

Although this puzzling situation might be related to observational bias and/or AGN variability (Hickox et al. 2013; Chen et al. 2013), a simple explanation is that the AGN activity is linked only to a particular type of star formation (Neistein \& Netzer 2013). Indeed, there is a growing observational evidence for two modes of star formation: a quiescent mode that takes place in most star forming galaxies with gas conversion timescales of $\simeq 1 \mathrm{Gyr}$, and the less common starburst mode acting on much shorter timescales of $\simeq 10^{7} \mathrm{yr}$ (e.g. Rodighiero et al. 2011; Lamastra et al. 2013). There is a strong theoretical argument indicating that the latter mode is the one related to the AGN activity, at least for the most luminous AGNs. In fact, to achieve high bolometric luminosities of $L_{\mathrm{bol}}=\eta \Delta M_{\mathrm{gas}} \mathrm{c}^{2} / \Delta t=$ $10^{46} \mathrm{erg} / \mathrm{s}$, typical of quasi stellar objects (QSO), in host galaxies with gas content of $5 \times 10^{8} M_{\odot}$, typical AGN activity times $\Delta t$ on the order of a few times $10^{7}$ yrs are required even for a large destabilized gas mass $\Delta M_{\text {gas }} \simeq M_{\text {gas }} / 5$ (where $\eta=0.1$ is commonly assumed for the radiative efficiency). This implies 
that fuelling a QSO requires the loss of a sizeable fraction of the disk angular momentum. This must happen on a timescale that is comparable to or shorter than the dynamical time of the galaxy. At present, galaxy merging seems to be the best (if not the only) mechanism with the above properties. Indeed, highresolution numerical simulations have shown the effectiveness of galaxy major merging in funnelling a large amount of gas onto the nuclear regions in a short timescale, and the resulting high gas density in the central region of the galaxy at the same time triggers starburst events (Hernquist 1989; Barnes \& Hernquist 1991, 1996; Mihos \& Hernquist 1994, 1996; Di Matteo et al. 2005; Cox et al. 2008). The connection between AGNs, starburst galaxies and galaxy mergers has several observational confirmations. Major mergers are associated with enhancements in star formation in local ultra luminous infrared galaxies (ULIRGs, Sanders \& Mirabel 1996; Elbaz et al. 2007), and at least some submillimeter galaxies (SMGs, Tacconi et al. 2008; Daddi et al. 2007, 2009; Engel et al. 2010; Fu et al. 2013). The fraction of galaxies hosting AGN activity is correlated to the IR luminosity (Kim et al. 1998; Veilleux et al. 1999; Tran et al. 2001; Alexander et al. 2008). Support for this scenario also comes from the signature of recent mergers in QSO hosts (see, e.g., Bennert et al. 2008; Treister et al. 2012). Based on the above evidence, semi-analytic models (SAMs) of BH and galaxy evolution assume galaxy major mergers as triggers for QSO activity (e.g. Kauffmann \& Haehnelt 2000; Menci et al. 2003, 2006; Croton et al. 2006; Bower et al. 2006; Hopkins et al. 2006; Monaco et al. 2007; Marulli et al. 2008; Somerville et al. 2008). For less luminous AGNs (Seyfert-like galaxies $L_{\text {bol }} \lesssim 10^{45} \mathrm{erg} / \mathrm{s}$ ), different fuelling mechanisms have been proposed in the literature. These include minor mergers, disk instabilities, the stochastic accretion of cold molecular clouds near the BH, and Bondi-Hoyle spherical accretion of hot gas from the diffuse atmosphere in the central bulge (e.g. Fanidakis et al. 2012; Hirschmann et al. 2012; Bournaud et al. 2011, 2012). These processes are often referred to as "secular processes" and their connection (if any) with the star formation of the host galaxies is less clear. Thus, a stronger correlation between SFR and AGN luminosity is expected for more luminous AGNs than for less luminous sources.

A direct approach to test the AGN-starburst-merger scenario is to identify a diagnostic that isolates the star formation directly related to the AGN luminosity and study its dependence on AGN luminosity. An effective tool for distinguish between quiescently star forming galaxies and starburst galaxies is provided by the scaling relation connecting the SFR with the total stellar mass. It has been shown that the former lie along a "main sequence" characterized by a typical redshift-dependent value of the SSFR (Brinchmann et al. 2004; Noeske et al. 2007; Elbaz et al. 2007; Daddi et al. 2007, 2009; Santini et al. 2009; Salim et al. 2007; Stark et al. 2009; González et al. 2011; Rodighiero et al. 2011), while the less numerous starburst population has higher SSFRs (e.g. Rodighiero et al. 2011). Thus the comparison between the observed distribution of AGN hosts in the SFR- $M_{*}$ plane and the observed fraction of starbursting hosts as a function of the AGN luminosity with those predicted by merger-driven models for starbursts and AGNs represent a test case for their basic assumption about the fuelling mechanism. In this paper we carry out these comparisons using a SAM of galaxy formation that includes a physical description of starburst and $\mathrm{BH}$ growth triggered by galaxy interaction during their merging histories (Menci et al. 2008) and a sample of X-ray selected AGNs in the XMM-COSMOS field in the redshift range $0.3<z<2$, and a sample of QSOs $\left(L_{\mathrm{bol}}>10^{46} \mathrm{erg} / \mathrm{s}\right)$ at $2<z<6.5$. Our SAM is ideally suited to this goal as it has been tested against the separation of starburst and quiescently star forming galaxies in the SFR- $M_{*}$ diagram as well as against several observational properties of the galaxy and AGN populations (Menci et al. 2004, 2005, 2006, 2008; Lamastra et al. 2010, 2013).

The paper is organized as follow. A description of the SAM is given in Sect. 2; in Sect. 3 we describe the properties of the AGN samples; in Sect. 4 we derive the predicted relation between the SFR and the AGN luminosity and the host galaxy stellar mass and the fraction of starbursting hosts as a function of AGN luminosity; conclusions follow in Sect. 5.

\section{The model}

We adopt the SAM described in details in Menci et al. (2004, 2005, 2006, 2008), which connects, within a cosmological framework, the baryonic processes (gas cooling, star formation, $\mathrm{BH}$ accretion, supernova and AGN feedback) to the merging histories of the dark matter (DM) halos. The latter, including the gradual inclusion of subhalos and their subsequent coalescence due to dynamical friction or binary aggregation, is computed adopting a Monte Carlo technique. The properties of the gas and stars contained in the DM halos are computed following the standard recipes commonly adopted in SAMs. Starting from an initial amount $m \Omega_{\mathrm{b}} / \Omega$ of gas at virial temperature of the galactic halos, we compute the mass $m_{\mathrm{c}}$ of cold baryons that are able to radiatively cool. The cooled gas mass $m_{\mathrm{c}}$ settles into a rotationally supported disk with radius $r_{\mathrm{d}}$ (typically ranging from 1 to $5 \mathrm{kpc}$ ), rotation velocity $v_{\mathrm{d}}$, and dynamical time $t_{\mathrm{d}}=r_{\mathrm{d}} / v_{\mathrm{d}}$, all computed after Mo et al. (1998). Stars form with a rate

$S F R_{q}=m_{\mathrm{c}} / \tau_{q}$,

where $\tau_{q}=q t_{\mathrm{d}}$, and $q$ is a model free parameter that is chosen to match the Kennicutt (1998) relation. In the following, we will refer to this mode of star formation as quiescent.

At each time step, the mass $\Delta m_{\mathrm{h}}$ returned from the cold gas content of the disk to the hot gas phase owing to the energy released by SNae following star formation is estimated from canonical energy balance arguments as $\Delta m_{\mathrm{h}}=E_{\mathrm{SN}} \epsilon_{0} \eta_{0} \Delta m_{*} / v_{\mathrm{c}}^{2}$, where $\Delta m_{*}$ is the stellar mass formed in the time step, $\eta_{0}$ is the number of SNe per unit solar mass (for a Salpeter initial mass function (IMF) $\left.\eta_{0}=6.5 \times 10^{-3} M_{\odot}^{-1}\right), E_{\mathrm{SN}}=10^{51} \mathrm{erg} / \mathrm{s}$ is the energy of ejecta of each SN, $v_{\mathrm{c}}$ the circular velocity of the galactic halo, and $\epsilon_{0}=0.01$ is a tunable efficiency for the coupling of the emitted energy with the cold interstellar medium.

\subsection{Starburst events and $\mathrm{BH}$ accretion triggered by galaxy interactions}

For a galactic halo with given circular velocity $v_{\mathrm{c}}$ inside a host halo with circular velocity $V$, the interactions occur at a rate

$\tau_{r}^{-1}=n_{T}(V) \Sigma\left(r_{\mathrm{t}}, v_{\mathrm{c}}, V\right) V_{\mathrm{rel}}(V)$,

where $n_{T}$ is the number density of galaxies in the host halo, $V_{\text {rel }}$ the relative velocity between galaxies, and $\Sigma \simeq \pi\left\langle r_{\mathrm{t}}^{2}+r_{\mathrm{t}}^{\prime 2}\right\rangle$ the cross section for such encounters, which is given by Saslaw (1985) in terms of the tidal radius $r_{\mathrm{t}}$ associated to a galaxy with given circular velocity $v_{\mathrm{c}}$ (see Menci et al. 2004).

The fraction $f$ of cold gas destabilized by the interactions has been worked out by Cavaliere \& Vittorini (2000) in terms of the variation $\Delta j$ of the specific angular momentum $j \approx G m / v_{\mathrm{d}}$ of the gas to read (Menci et al. 2004):

$f \approx \frac{1}{2}\left|\frac{\Delta j}{j}\right|=\frac{1}{2}\left\langle\frac{m^{\prime}}{m} \frac{r_{\mathrm{d}}}{b} \frac{v_{\mathrm{d}}}{V_{\mathrm{rel}}}\right\rangle$, 
where $b$ is the impact parameter, evaluated as the greater of the radius $r_{\mathrm{d}}$ and the average distance of the galaxies in the halo, $m^{\prime}$ is the mass of the partner galaxy in the interaction, and the average runs over the probability of finding such a galaxy in the same halo where the galaxy with mass $m$ is located. The pre-factor accounts for the probability $1 / 2$ of inflow rather than outflow related to the sign of $\Delta j$. AGN and starburst events are triggered by all galaxy interactions including major mergers ( $\left.m \simeq m^{\prime}\right)$, minor mergers $\left(m \ll m^{\prime}\right)$, and by fly-by events. We assume that in each interaction $1 / 4$ of the destabilized fraction $f$ feeds the central $\mathrm{BH}$, while the remaining fraction feeds the circumnuclear starbursts (see Sanders \& Mirabel 1996). Thus, the SFR in the nuclear region due to interaction-driven burst is given by

$$
S F R_{b}=(3 / 4) f m_{\mathrm{c}} / \tau_{b} \text {. }
$$

Here the timescale $\tau_{b}$ is assumed to be the crossing time for the destabilized cold gas component $\left(t_{\mathrm{d}}\right)$. This adds to the quiescent SFR given in Eq. (1).

The accreted mass $\Delta m_{a c c}=(1 / 4) f m_{\mathrm{c}}$ into the BH powers the AGN emission with bolometric luminosity

$L_{\mathrm{bol}}=\frac{\eta c^{2} \Delta m_{\mathrm{acc}}}{\tau_{\mathrm{AGN}}}$

The duration of an accretion episode, i.e., the timescale for the QSO or AGN to shine, is $\tau_{\mathrm{AGN}}=t_{\mathrm{d}}$. We adopt an energyconversion efficiency $\eta=0.1$ (see Yu \& Tremaine 2002), and derive the X-ray luminosities in the $2-10 \mathrm{keV}$ band $\left(L_{X}\right)$ from the bolometric correction given in Marconi et al. (2004)

\subsection{AGN feedback and column density of absorbing gas}

Our SAM includes a detailed treatment of AGN feedback that is directly related to the impulsive, luminous AGN phase (Menci et al. 2006, 2008). This is based on expanding blast wave as a mechanism to propagate outwards the AGN energy injected into the interstellar medium at the center of galaxies. The injected energy is taken to be proportional to the energy radiated by the AGN, $E=\epsilon_{\mathrm{AGN}} \eta c^{2} \Delta m_{\mathrm{acc}}$, where $\epsilon_{\mathrm{AGN}}=5 \times 10^{-2}$ is the value of the energy feedback efficiency for coupling with the surrounding gas. All the shock properties depend on this quantity. The AGN emission is absorbed by the unperturbed amount of gas in the galaxy disk outside the shock. To calculate the neutral hydrogen column densities $\left(N_{\mathrm{H}}\right)$ of the unshocked absorbing gas we extract a random line-of-sight angle $\theta$, which defines the disk inclination to the observer. At time $t$ within the interval $\tau_{\text {AGN }}$ corresponding to the active AGN, we compute $N_{\mathrm{H}}$ corresponding to the gas outside the shock position along the selected line of sight as

$N_{\mathrm{H}}=\int_{R_{\mathrm{s}}(t)}^{h / \sin \theta} \rho(r) \mathrm{d} l$,

where $R_{\mathrm{S}}(t)$ is the shock position after a time $t$ from an AGN outburst, $h=r_{\mathrm{d}} / 15$ is the disk thickness (see Narayan \& Jog 2002), and $\rho$ is the density distribution of the unperturbed gas for which we assumed the form $\rho=\rho_{0} \exp \left(-r / r_{\mathrm{d}}\right)$ (where $r$ is the distance from the center of the galaxy) with a cut-off in the direction perpendicular to the disk at $r=h$. The density distribution is normalized so as to recover the total gas mass $m_{\mathrm{c}}$ when integrated over the disk volume (see Menci et al. 2008).

The model has been tested against several observational properties of the galaxy and AGN populations such as the evolution of the galaxy and AGN luminosity functions in different bands, the local $M_{\mathrm{BH}}-M_{*}$ relation, the galaxy bimodal color distribution, the Tully-Fisher relation, the fraction of obscured AGN as a function of luminosity and redshift, and the relative contribution of starburst and main sequence galaxies to the cosmic SFR density (Menci et al. 2004, 2005, 2006, 2008; Lamastra et al. 2010, 2013).

\section{Data set}

Since we aim at separating the burst mode of star formation from the quiescent one through the value of the SSFR we need AGN samples with measured values of SFR and stellar mass.

The unprecedented wide and deep multiwavelength coverage of the COSMOS field makes it possible to derive the total stellar mass, as well as the other stellar parameters, in statistically representative samples of galaxies through the spectral energy distribution (SED) fitting technique. In our analysis we use a sample of X-ray selected AGNs from the XMM-Newton survey of the COSMOS field in the redshift range $0.3<z<2$ (Scoville et al. 2007). The XMM-COSMOS catalog has been presented by Cappelluti et al. (2009), while the optical identifications and multiwavelength properties have been discussed by Brusa et al. (2010). The X-ray luminosities in the $2-10 \mathrm{keV}$ band $\left(L_{X}\right)$ are derived by Mainieri et al. (2007, 2011). Whenever possible, the de-absorbed X-ray luminosities are determined with a proper spectral analysis. Otherwise, the absorbing column densities are derived from the hardness ratio assuming a given photon index.

The stellar masses of the XMM-COSMOS AGN host galaxies are derived by Santini et al. (2012) and Bongiorno et al. (2012) by fitting the observed SEDs with a two component model based on a combination of AGN and host galaxy templates. Bongiorno et al. (2012) used the SED fitting technique in the optical/IR bands also to derive the SFRs. This procedure relies on measurement of the UV light emission from young stars corrected for dust extinction. As discussed by the authors, these SFRs are reliable only for obscured AGNs where the UV range is clean of AGN contamination.

Based on the assumption that the AGN contamination in FIR emission is not dominant over the emission of cold dust heated by young stars, we derive the SFRs of XMM-COSMOS AGNs from the dust thermal emission at these wavelenghts. We use the $160 \mu \mathrm{m}$ data collected by the PACS instrument (Poglitsch et al. 2010) on board the Herschel Space Observatory (Pilbratt et al. 2010), as part of the PACS Evolutionary Probe (Lutz et al. 2011) survey. The fraction of XMM-COSMOS AGNs in the redshift interval $0.3<z<2$ detected at $160 \mu \mathrm{m}$ is $20 \%$. A number of previous studies support this assumption. Indeed, many authors (e.g. Schweitzer et al. 2006; Netzer et al. 2007; Lutz et al. 2008) find a strong correlation between FIR luminosity and SFR tracers, such as polycyclic aromatic hydrocarbon emission features, both in local and high redshift bright AGNs. However, the $160 \mu \mathrm{m}$ band corresponds to $53-123 \mu \mathrm{m}$ rest-frame wavelength band in the redshift interval considered in this work, thus at high redshift we are sensitive to warmer dust emission. Nevertheless, this band is not strongly affected by the AGN emission as suggested by the study of Rosario et al. (2012) (see also Santini et al. 2012). In the same redshift interval, they compared the FIR $100 \mu \mathrm{m}$ to $160 \mu \mathrm{m}$ color of the XMM-COSMOS AGNs with that of inactive mass-matched galaxies finding no significant difference between the two samples. Since the typical AGN luminosity of flux-limited samples like those from XMM-COSMOS increases toward higher redshifts, while the $160 \mu \mathrm{m}$ band traces increasingly shorter rest-frame wavelengths, we cannot, however, exclude a minor contribution from the AGN emission in the most distant sources used in this work. 
To estimate the SFR the flux at $160 \mu \mathrm{m}$ is fitted with the Dale \& Helou (2002) template library to derive the total IR luminosity $\left(L_{\mathrm{IR}}\right)$ integrated between $8 \mu \mathrm{m}$ and $1000 \mu \mathrm{m}$. The latter is converted into SFR using the relation $S F R\left[M_{\odot} / \mathrm{yr}\right]=1.7 \times$ $10^{-10} L_{\mathrm{IR}}\left[L_{\odot}\right]$ (Kennicutt 1998).

All the SFRs and stellar masses used in this work are computed using a Salpeter IMF.

To extend the analysis at higher redshifts and AGN luminosities, we collect from the literature 28 QSOs with $L_{\text {bol }} \geq$ $10^{46} \mathrm{erg} / \mathrm{s}$ (corresponding to $L_{\mathrm{X}} \gtrsim 10^{44.5} \mathrm{erg} / \mathrm{s}$ applying the bolometric correction of Marconi et al. 2004) at $2<z<6.5$ (Polletta et al. 2008, 2011; Lacy et al. 2011; Wang et al. 2010, 2013; Solomon \& Vanden Bout 2005; Coppin et al. 2008; Shields et al. 2006; Maiolino et al. 2007; Gallerani 2012). In this sample, only three QSOs have estimates of the host galaxy stellar mass and SFR from SED fitting. For the other 21 sources we infer the stellar masses from observations of molecular carbon monoxide (CO) emission lines. Indeed, these observations provide valuable constraints on the gas content and dynamical state of these systems. Under the assumption that the gas is driven by gravity and is approximately virialized, the dynamical mass of the host galaxy can be estimated as $M_{\text {dyn }}=R v^{2} / G \sin ^{2} i$, where $R$ is the disk radius, $v$ is the circular velocity at the outer disk radius which is measured from the $\mathrm{CO}$ line width, and $i$ the inclination angle of the gaseous disk. The main uncertainty of the dynamical mass is due to the unknown inclination angle $i$. We derive the mean value of the dynamical mass assuming randomly oriented disks with respect to the sky plane as:

$\left\langle M_{\mathrm{dyn}}\right\rangle=\frac{\int_{i_{\min }}^{i_{\max }} \frac{R v^{2}}{G \sin ^{2} i} \sin i \mathrm{~d} i}{\int_{i_{\min }}^{i_{\max }} \sin i \mathrm{~d} i}$

where $i_{\min }$ is the minimum disk inclination angle obtained by setting $M_{*}=M_{\text {dyn }}-M_{\text {gas }}<10^{13} M_{\odot}$, and $i_{\max }$ is the maximum inclination angle derived by setting $M_{\text {dyn }}>M_{\text {gas }}$. We estimate the molecular gas $\left(\mathrm{H}_{2}\right)$ mass from the $\mathrm{CO}$ line luminosity assuming a $\mathrm{CO}$ intensity-to-gas mass conversion factor of $\alpha_{\mathrm{CO}}=M_{\text {gas,mol }} / L_{\mathrm{CO}(1-0)}=0.8 M_{\odot}\left(\mathrm{K} \mathrm{km} \mathrm{s}^{-1} \mathrm{pc}^{2}\right)^{-1}$, as it is commonly assumed for ULIRGs and QSO host galaxies (e.g. Solomon \& Vanden Bout 2005; Wang et al. 2010). Then we infer the stellar masses as $M_{*}=M_{\mathrm{dyn}}-M_{\mathrm{gas}, \mathrm{mol}}$. We here assume negligible atomic gas (HI) contribution to the total gas mass of high- $z$ QSO hosts. Observational evidences (e.g. Daddi et al. 2010a; Tacconi et al. 2010; Geach et al. 2011) and theoretical arguments (Blitz \& Rosolowsky 2006; Obreschkow \& Rawlings 2009) indicate that the $\mathrm{H}_{2} / \mathrm{HI}$ fraction increases with redshift, making us confident of the assumption adopted. By comparing the relation between $L_{\mathrm{CO}}$ and the luminosity at $42.5-122.5 \mu \mathrm{m}$ rest-frame wavelength band $\left(L_{\mathrm{FIR}}\right)$ of the CO-detected QSOs with that of galaxies without a luminous AGN, Riechers (2011) found that $L_{\mathrm{FIR}}$ in these QSO is dominated by dust-reprocessed emission from young stars in the host galaxy, rather than the AGN. Following Riechers (2011), we derive their SFRs from $L_{\mathrm{FIR}}$ under the conservative assumptions that $L_{\mathrm{IR}} \simeq L_{\mathrm{FIR}}$, and that $10 \%$ of $L_{\mathrm{FIR}}$ is actually powered by the AGN and not the starburst.

We use the same relation to derive the SFR also for the remaining 4 sources in this sample. To estimate their stellar masses we use the angle-corrected dynamical masses within the singly ionized carbon ([C II]) emitting region from Wang et al. (2013), and the gas masses derived from CO observations (Wang et al. 2010, 2013) as described above.

We also include in this sample HS1700+6416 $\left(L_{X}=4 \times\right.$ $10^{45} \mathrm{erg} / \mathrm{s}$, Lanzuisi et al. 2012) for which we measure an SFR

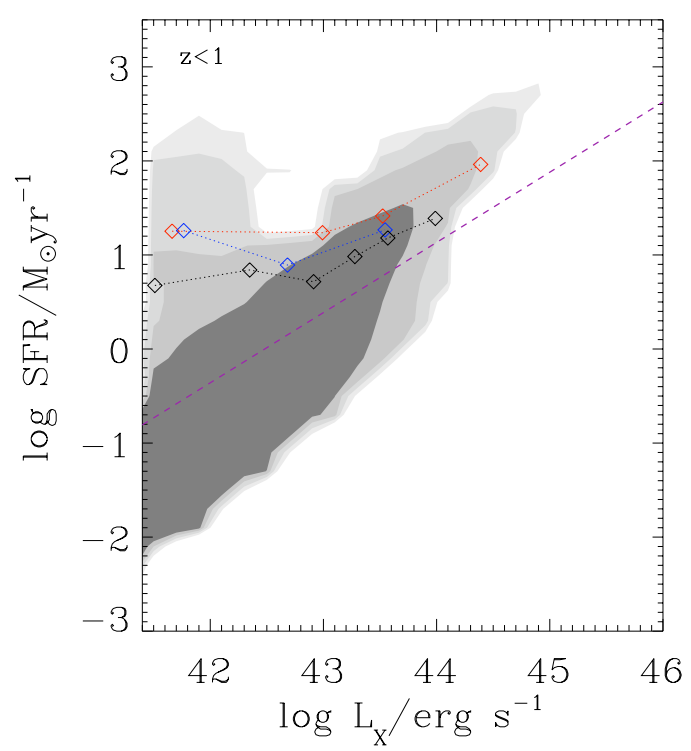

Fig. 1. SFR versus $L_{\mathrm{X}}$ at $z<1$. The four filled contours correspond to equally spaced values of the density (per $\mathrm{Mpc}^{3}$ ) of model AGNs in logarithmic scale: from $10^{-7}$ for the lightest filled region to $10^{-4}$ for the darkest. The dashed line is the relation obtained by Netzer (2009). Observational results from Rosario et al. (2012) at $z<0.3$, $0.2<z<0.5,0.5<z<0.8$, are shown in black, blue and red dotted lines, respectively.

of $2454 M_{\odot} / \mathrm{yr}$ and a stellar mass of $3 \times 10^{11} M_{\odot}$ from SED fitting (Bongiorno et al., in prep.).

\section{Results}

\subsection{The $S F R-L_{X}$ relation}

We start by showing in Fig. 1 the SFR- $L_{X}$ relation of model AGNs at $z<1$. Given the modest redshift evolution of the predicted SFR- $L_{\mathrm{X}}$ relation in this redshift range, we represent the whole redshift interval on the same SFR- $L_{X}$ plane to provide an overview of the trend of SFR as a function of $L_{X}$. The plotted SFR is the total SFR of the host galaxy which is given by the sum of the quiescent and burst component of star formation (Eqs. (1) and (4)). Since the accretion rate onto the BHs is correlated only to the latter mode of star formation we find a strong correlation between SFR and $L_{X}$ for luminous AGNs, and a more scattered relation for the less luminous sources owing to the larger contribution of the quiescent component of star formation to the total SFR of the galaxy in these objects. In Fig. 1 we also show the observed relations for local optically-selected AGNs (Netzer 2009) and for X-ray selected AGNs at $z<0.8$ (Rosario et al. 2012). The data points from Rosario et al. (2012) do not represent individual objects but mean trends that come from combining fluxes from detections and stacks of undectected sources in the Herschel-PACS bands in bins of redshift and X-ray luminosity. Following Santini et al. (2012), we convert the rest frame luminosity at a wavelength of $60 \mu \mathrm{m}$ (as presented in their works) into $L_{\mathrm{IR}}$ by linearly fitting the values of $v L_{v}(60 \mu \mathrm{m})$ and $L_{\mathrm{IR}}$ predicted by the Dale \& Helou (2002) templates $\left(\log v L_{v}(60 \mu \mathrm{m})=1.07 \times \log L_{\mathrm{IR}}-1.18\right.$, we find a very similar relation using the Chary \& Elbaz 2001 template library). We then compute the SFR by using the relation given in Sect. 3. A detailed comparison with the observational results is beyond the scope of this paper since this requires an accurate estimate of the different selection criteria of the AGN samples. 
Here we note that pinning down the AGN triggering mechanism from the SFR- $L_{X}$ relation is a critical issue. On the observational side, the observed relation is still uncertain due to observational bias and instrumental limitation in sensitivity. On the theoretical side, even a model based on galaxy interactions as triggers of AGN and starburst activities, predicts a large scatter $(\simeq 3$ orders of magnitude) at low AGN luminosities owing to the pollution of the global star formation by the quiescent mode.

\subsection{The starburstiness $-L_{x}$ relation}

A step forward in our understanding of AGN triggering mechanisms can be done by comparing the AGN luminosity with the starburstiness $R_{\mathrm{SB}}=S S F R / S S F R_{\mathrm{MS}}$ of the host galaxy (Elbaz et al. 2011), where the subscript MS indicates the typical value for main sequence galaxies. The quantity $R_{\mathrm{SB}}$ measures the excess or deficiency in SSFR of a star forming galaxy in terms of its distance from the galaxy main sequence. In our previous paper (Lamastra et al. 2013) we showed that the correlation between SFR and $M_{*}$ of model galaxies on the main sequence is determined by galaxies dominated by the quiescent component of star formation $\left(S F R_{\mathrm{q}}>S F R_{\mathrm{b}}\right)$, while galaxies dominated by the burst component of star formation $\left(S F R_{b}>S F R_{q}\right)$ have higher values of $R_{\mathrm{SB}}$. Interestingly, we found that the criterion $R_{\mathrm{SB}}>4$, which is commonly assumed to observationally classified starburst galaxies (e.g. Rodighiero et al. 2011; Sargent et al. 2012), works well in filtering out quiescently star forming galaxies. Since the more luminous the AGN the larger the burst component of star formation, interaction-driven models for AGNs predict a strong correlation between $R_{\mathrm{SB}}$ and the AGN luminosity. Such a strong luminosity dependence of $R_{\mathrm{SB}}$ is illustrated in Fig. 2 (left panels), where we show with the contours the average values of the AGN X-ray luminosity as a function of $R_{\mathrm{SB}}$ and $M_{*}$ in three different redshift bins: $0.3<z<0.9,0.9<z<1.3$, and $1.3<z<2$. The figure illustrates that indeed for host galaxies with large values of $R_{\mathrm{SB}}$ high AGN luminosities are expected. An immediate implication of the above is that the fraction of AGN hosts with SSFR above the starburst threshold $\left(R_{\mathrm{SB}}>4\right)$ increases with AGN luminosity.

To test this prediction we compare in Fig. 2 the luminosity and density distributions in the $R_{\mathrm{SB}}-M_{*}$ diagram of model AGNs (in the central panels the color coding identifies the volume density) with that of X-ray selected AGNs in the XMM-COSMOS field (Sect. 3). We restrict the analysis to the most luminous sources with $L_{X} \geq 10^{44} \mathrm{erg} / \mathrm{s}$. For luminous AGNs the accretion time $\left(\tau_{\mathrm{AGN}} \simeq 10^{7} \mathrm{yrs}\right)$ is small when compared with the typical lifetime of the SFR indicators based on UV and IR emissions that last $\sim 10^{8}$ yrs (Neistein \& Netzer 2013). The latter time is also longer than the gas depletion time expected from AGN feedback models based on expanding blast wave. Thus, even if the AGN feedback immediately follows the BH accretion, the signature of star formation in luminous AGNs should be detected even if the galactic gas has been swept out by the AGN feedback.

Hierarchical clustering models, connecting the properties of galaxies to their merging histories, reproduce the slope and the scatter of the SSFR- $M_{*}$ relation; however, they under-predict its normalization at $z \lesssim 2$ (Daddi et al. 2007; Davé 2008; Fontanot et al. 2009; Damen et al. 2009; Santini et al. 2009; Lin et al. 2012; Weinmann et al. 2011; Lamastra et al. 2013). A possible theoretical explanation of this mismatch is that the amount of cold gas in galaxy disks predicted by these models underestimates the real values. In this analysis we normalize both the model and observed SSFRs to their main sequence values. Both in the model and in the data we obtain SSFR $_{M S}$ that depends on redshift and stellar mass. To derive a characteristic $\mathrm{SSFR}_{\mathrm{MS}}$ at a given redshift and stellar mass, for model galaxies we separately $\mathrm{fit}^{1}$ the peaks of the SFR distributions as a function of the stellar mass with the relation $\log S F R=a \log M_{*}+b$ in each individual redshift bin. We found values for $(a, b)$ equal to $(0.91,-9.02),(0.96,-9.31)$, and $(0.93-8.76)$ in the three redshift bins from $z=0.3$ to $z=2$, respectively. For the observational data we use the best-fit of the galaxy main sequences obtained by Santini et al. (2009) in similar redshift intervals. These relations have slopes flatter than those obtained for model galaxies ranging from 0.65 to 0.85 .

As it can be seen in Fig. 2 (central panels) the model predicts that low mass galaxies $\left(M_{*} \lesssim 10^{10} M_{\odot}\right)$ hosting bright AGNs $\left(L_{\mathrm{X}} \geq 10^{44} \mathrm{erg} / \mathrm{s}\right)$ are predicted to populate the starburst region $\left(R_{\mathrm{SB}}>4\right)$, while higher stellar mass hosts mainly populate the main sequence $\left(1 / 4<R_{\mathrm{SB}}<4\right)$ and the passive areas $\left(R_{\mathrm{SB}}<1 / 4\right)$. The effectiveness of the $R_{\mathrm{SB}}>4$ criterion in filtering out quiescently star forming galaxies can be inferred from the right panels of Fig. 2, which show separately the starburstiness distributions of AGN hosts dominated by the quiescent component of star formation, and of AGN hosts dominated by the burst component of star formation. Indeed, all the galaxies in the starbust region are dominated by the burst component of star formation. The main sequence region is nearly equally populated by AGN hosts with $S F R_{b}>S F R_{q}$ and with $S F R_{q}>S F R_{b}$, while the passive area is populated by massive galaxies dominated by the burst component of star formation. Such massive hosts formed from biased, high density regions of the primordial density field where the frequent high- $z$ interactions rapidly convert the cold gas reservoir into stars at early cosmic epochs, leaving only a residual fraction of cooled gas available for the star formation at $z \lesssim 2$. These massive galaxies represent only the $1 \%$ of the $L_{X} \geq 10^{44} \mathrm{erg} / \mathrm{s}$ AGN hosts at $z>0.9$, at lower redshift this fraction increases up to $10 \%$.

We also show in Fig. 2 the model predictions obtained by selecting from the model AGNs with $L_{\mathrm{X}} \geq 10^{43.8} \mathrm{erg} / \mathrm{s}$ (dotted contours in the central panels). Indeed, the uncertainty related to the estimate of the intrinsic AGN X-ray luminosity due to the uncertainty in the bolometric correction ${ }^{2}$ and to uncertainty in AGN absorbing column density measurements (especially from hardness ratio) can affect the comparison between the model and the data. We note that a larger discrepancy in the luminosity distribution in the $R_{\mathrm{SB}}-M_{*}$ diagram between the data and the model is observed for the obscured AGN sample (see left panels of Fig. 2).

By comparing the density distribution of model AGNs with that of the XMM-COSMOS AGNs with Herschel observations (Fig. 2, central panels), we find that all FIR-detected AGNs lie in the predicted confidence region represented by the contour plots. However, this comparison is hampered by the Herschel detection limit in the COSMOS field (corresponding to SFR limits of $\sim 6 M_{\odot} / \mathrm{yr}$ at $z=0.3$ and $\sim 400 M_{\odot} / \mathrm{yr}$ at $z=2$ ) that allows to estimate only SFR upper limits for a large number of the sources. For about 30\% (59/196) of the Herschel-undetected sources SFR estimates from SED fitting in the optical/IR band are available. Only for a very small fraction of these sources (2/59) $160 \mu \mathrm{m}$ upper limits are not consistent with the SED-based estimates. By comparing the model predictions with the obscured AGN sample with SED-based SFRs we do not find an exact overlap

\footnotetext{
We use the sixlin IDL routine.

2 For model AGNs we use the luminosity-dependent bolometric correction factor given in Marconi et al. (2004) to derive X-ray luminosities in the $2-10 \mathrm{keV}$ band from bolometric luminosities.
} 


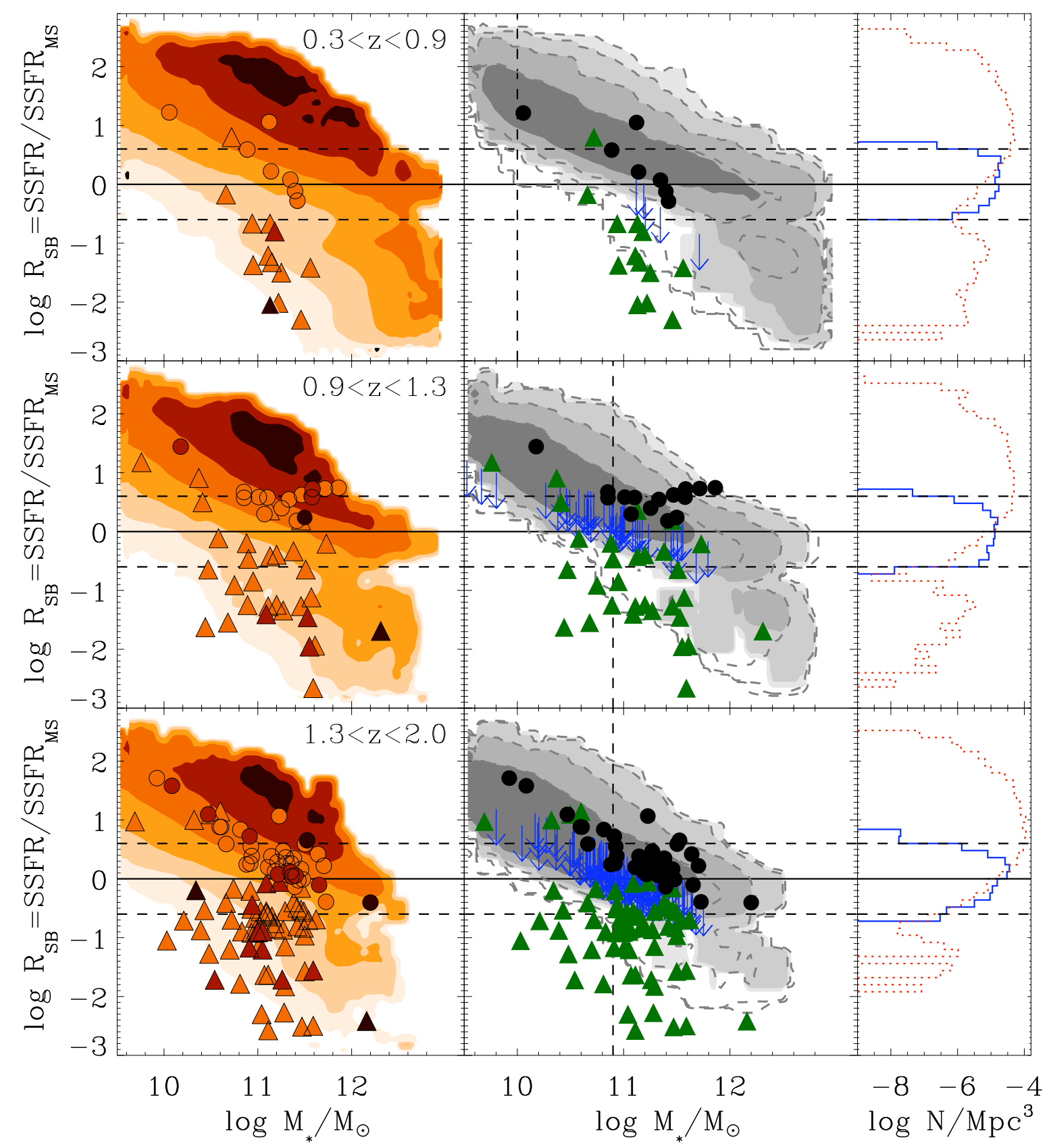

Fig. 2. Left and central panels: starburstiness $R_{\mathrm{SB}}$ as a function of $M_{*}$ in three redshift bins. The filled contours in the left panels correspond to the predicted average values of the AGN X-ray luminosity for bins of different $M_{*}$ and $R_{\mathrm{SB}}$. The luminosity values are equally spaced in logarithmic scale from $L_{\mathrm{X}}=10^{42.5} \mathrm{erg} / \mathrm{s}$ for the lightest filled region to $L_{\mathrm{X}}=10^{45} \mathrm{erg} / \mathrm{s}$ for the darkest. The filled (dotted) contours in the central panel correspond to equally spaced values of the density (per $\left.\mathrm{Mpc}^{3}\right)$ of model AGNs with $L_{\mathrm{X}} \geq 10^{44} \mathrm{erg} / \mathrm{s}\left(L_{\mathrm{X}} \geq 10^{43.8} \mathrm{erg} / \mathrm{s}\right)$ in logarithmic scale: from $10^{-9}$ for the lightest filled region to $10^{-6}$ for the darkest. The data points indicate the XMM-COSMOS AGNs with $L_{\mathrm{X}} \geq 10^{44} \mathrm{erg} / \mathrm{s}$. Circles and arrows indicate AGNs with SFR derived from $L_{\mathrm{FIR}}$, while triangles indicate AGNs with SFR derived from SED fitting. Circles and triangles are color coded according to their X-ray luminosity in the left panels. Solid lines show the position of the galaxy main sequence, while dashed lines denote the limits of the starburst and passive areas, defined as $R_{\mathrm{SB}}>4$ and $R_{\mathrm{SB}}<1 / 4$, respectively. Vertical dashed lines indicate the stellar mass limits adopted in deriving the fraction of AGN hosted in starburst galaxies in Sect. 4.2. Right panels: starburstiness distribution of model AGNs. The solid histograms refer to galaxies dominated by the quiescent mode of star formation $\left(S F R_{q}>S F R_{b}\right)$, while the dotted histograms refer to galaxies dominated by the burst component of star formation $\left(S F R_{b}>S F R_{q}\right)$. Solid and dashed lines as in the left panels.

with the confidence regions predicted by the model in the passive areas. Indeed, the model predicts that the hosts of luminous AGNs with low star formation are more massive than observed, especially in the lowest redshift bin. This may be related to an incompleteness in our treatment of the AGN feedback which is a mechanism of suppression of the cooling in massive halos. Such feedback must be still at work at low redshift to continuously suppress star formation in massive halos at $z<1$. In fact, such long-standing problem of the hierarchical scenario of galaxy formation leads to the over-prediction of high mass galaxies in the local Universe as shown by comparing ours and other SAMs with the observed stellar mass function (e.g. Menci et al. 2005; Fontanot et al. 2009; Guo et al. 2011, but see Bernardi et al. 2013; Mitchell et al. 2013).

However, the mismatch between the data and the model at low SSFR values could be at least partially explained by the 
systematics affecting the SFR indicators. Bongiorno et al. (2012) compared the SFRs computed using FIR data and those computed with the SED fitting for the AGNs in the COSMOS field finding that the SFR computed from the SED are systematically lower than the one derived from the FIR (see Fig. B1 of Bongiorno et al. 2012). They concluded that this discrepancy is the result of a combination of two effects: (i) the tendency for the SED fit to overestimate the AGN emission component; (ii) the FIR overestimation of the SFR, especially at high AGN luminosities and low SFR, where the AGN contamination in the IR band is not negligible.

To study the dependence of the starburstiness of the host galaxy on the AGN luminosity, we estimate the fraction $f_{\text {bursty }}=$ $N_{\mathrm{AGN}}^{\mathrm{SB}} / N_{\mathrm{AGN}}$ of AGN host galaxies with $R_{\mathrm{SB}}>4$ relative to the total number of AGN hosts as a function of $L_{\mathrm{X}}$. The predictions from the model for different host galaxy stellar masses are shown as lines in Fig. 3. As expected, a strong correlation is predicted by the model for high AGN luminosities $\left(L_{X} \geq 10^{44} \mathrm{erg} / \mathrm{s}\right)$. The fraction $f_{\text {bursty }}$ is predicted to increase rapidly from $\lesssim 0.2$ at $L_{\mathrm{X}} \lesssim 10^{44} \mathrm{erg} / \mathrm{s}$ to $\gtrsim 0.9$ at $L_{\mathrm{X}} \gtrsim 10^{45} \mathrm{erg} / \mathrm{s}$.

In this figure we compare the model predictions with the observational results obtained from the FIR-based SFRs and the stellar masses derived by Santini et al. (2012). The estimate of $f_{\text {bursty }}$ from FIR data is prevented by the large number of Herschel undetected sources. However, we note that, in each redshift bin above a given stellar mass all AGNs in the starburst region are detected by Herschel (see Fig. 2). Thus, above these stellar masses we can properly estimate $N_{\mathrm{AGN}}^{\mathrm{SB}}$ and hence $f_{\text {bursty }}$. These stellar mass limits depend primarly on the instrumental sensitivity which corresponds to larger SFR detection limits at higher redshifts, and on the evolution of the galaxy main sequence. For the evolution adopted in this paper they correspond to $M_{*} \geq 10^{10} M_{\odot}$ at $0.3<z<0.9$, and to $M_{*} \geq 10^{10.9} M_{\odot}$ in the higher redshift bins. The fractions $f_{\text {bursty }}$ are computed in $L_{\mathrm{X}}$ intervals optimized to have roughly the same number of sources in each bin. The results are shown in Fig. 3, where, in each luminosity interval, the plotted value of $L_{X}$ is the median value in the bin and $1 \sigma$ uncertainties are derived through binomial statistics. For $L_{\mathrm{X}} \lesssim 10^{44.5} \mathrm{erg} / \mathrm{s}$ the data are in reasonable good agreement with the model predictions, except for AGNs with $L_{\mathrm{X}} \simeq 10^{44.1} \mathrm{erg} / \mathrm{s}$ at $0.9<z<1.3$ for which the model predicts a lower value of $f_{\text {bursty }}$ than that observed. The measurements of the SFR and stellar mass of AGN hosts in larger area surveys are necessary to probe with the required statistics the high-luminosity range $\left(L_{\mathrm{X}} \geq 10^{44.5} \mathrm{erg} / \mathrm{s}\right)$ where the strongest dependence of $f_{\text {bursty }}$ on $L_{\mathrm{X}}$ is expected.

We also tested the robustness of our results by adopting different galaxy main sequences for the observational data. By adopting the galaxy main sequence from Whitaker et al. (2012), and an SFR $_{M S}$ that varies with stellar mass with a slope of 0.8 (e.g. Rodighiero et al. 2011) and evolves with time as $(1+z)^{2.95}$ (e.g. Pannella et al. 2009; Elbaz et al. 2011; Magdis et al. 2012), we found consistent results within the errors, except at $0.9<z<$ 1.3 once the latter parametrization is assumed. For this redshift range we found $f_{\text {bursty }}=0$ in each luminosity bin. This can be explained by the steeper slope of the galaxy main sequence that corresponds to larger $\mathrm{SSFR}_{\mathrm{MS}}$ for galaxies with $M_{*} \gtrsim 10^{10} M_{\odot}$.

\subsubsection{The role of obscuration}

In the previous section we showed that the Herschel sensitivity in the COSMOS field allows us to derive $f_{\text {bursty }}$ only for host galaxies with large stellar masses. In order to extend this analysis
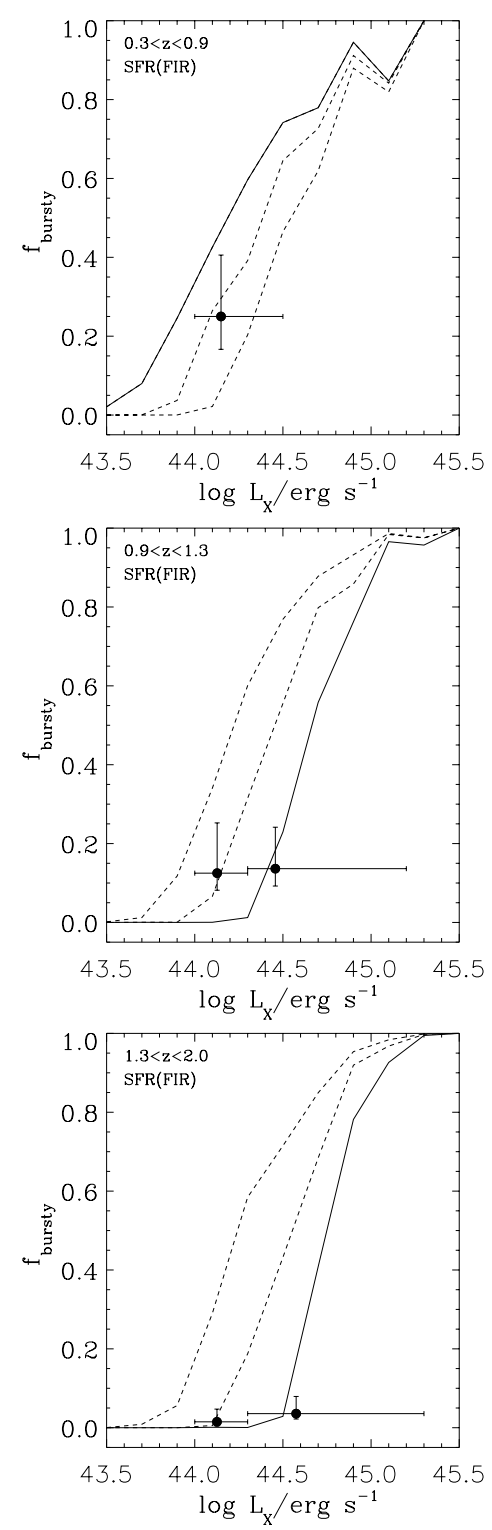

Fig. 3. $f_{\text {bursty }}$ versus $L_{X}$ in three redshift bins. The lines show the model predictions obtained by selecting AGNs with $M_{*} \geq 10^{10} M_{\odot}, M_{*} \geq$ $10^{10.5} M_{\odot}$, and $M_{*} \geq 10^{10.9} M_{\odot}$ from left to right. Solid lines indicate the stellar mass limits used to derive the observational fractions. The data points are derived using the FIR-based SFRs and $M_{*}$ was derived by Santini et al. (2012). The plotted value of $L_{X}$ is the median value for sources in each luminosity bin. Vertical error bars indicate the $1 \sigma$ binomial uncertainties. Horizontal error bars indicate the luminosity bin sizes which are optimized to have roughly the same number of sources in each bin.

to lower stellar mass hosts, we derive $f_{\text {bursty }}$ using the SFRs derived from the SED fitting technique. This allow us to derive an independent estimate of $f_{\text {bursty. }}$. However, the SED-based SFRs restricts the analysis only to obscured AGNs (see Sect. 3). Using the SFRs and the stellar masses derived by Bongiorno et al. (2012) we estimate $f_{\text {bursty }}$ as a function of $L_{X}$ (in the usual three redshift bins) as computed in the previous section. The results are shown with the data points in Fig. 4. These fractions remain almost unchanged if the different parametrizations of the galaxy main sequences described in the previous section are adopted.

As a comparison, we show the model predictions obtained by selecting only obscured AGNs. We use the canonical absorbing column density $N_{\mathrm{H}} \geq 10^{22} \mathrm{~cm}^{-2}$ to select from the model 

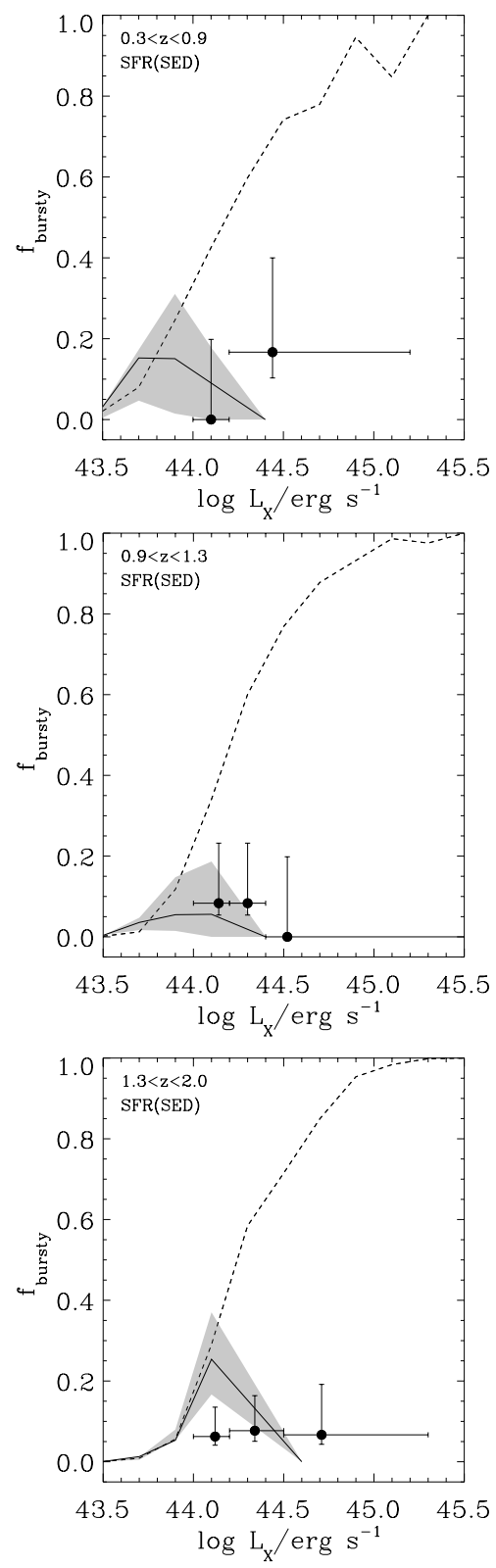

Fig. 4. $f_{\text {bursty }}$ versus $L_{\mathrm{X}}$ in three redshift bins. The solid lines show the model predictions obtained by selecting obscured AGNs with $N_{\mathrm{H}}>$ $10^{22} \mathrm{~cm}^{-2}$ and $M_{*} \geq 10^{10} M_{\odot}$. The upper and the lower envelopes of the shaded regions show $f_{\text {bursty }}$ corresponding to the selection $N_{\mathrm{H}} \geq$ $10^{21.8} \mathrm{~cm}^{-2}$ and $N_{\mathrm{H}} \geq 10^{22.2} \mathrm{~cm}^{-2}$, respectively. The dashed lines show the predictions obtained by selecting obscured and unobscured AGNs with $M_{*} \geq 10^{10} M_{\odot}$. The data points are derived using the SED-based SFRs and $M_{*}$ as computed by Bongiorno et al. (2012). The plotted value of $L_{\mathrm{X}}$ is the median value for sources in each luminosity bin. Vertical error bars indicate the $1 \sigma$ binomial uncertainties. Horizontal error bars indicate the luminosity bin sizes which are optimized to have roughly the same number of sources in each bin.

obscured AGNs. However, the exact values of $f_{\text {bursty }}$ predicted by the model depend on the $N_{\mathrm{H}}$ threshold adopted as it is indicated by the upper and the lower envelopes of the shaded regions which show the fractions obtained by selecting AGNs with $N_{\mathrm{H}} \geq 10^{21.8} \mathrm{~cm}^{-2}$ and $N_{\mathrm{H}} \geq 10^{22.2} \mathrm{~cm}^{-2}$, respectively.

We compare the model predictions with the observations, with the caveat that in the model the obscuration is associated only to cold gas in the galaxy disk, while the observational classification is based on both nuclear and galactic obscuration (see
Bongiorno et al. 2012, for the details about the AGN classification). The model predictions are in reasonable good agreement with the observational data in the luminosity ranges where obscured AGNs are predicted. However, the observations indicate the presence of obscured AGNs also at higher luminosities than those predicted.

A striking feature of the model is that at high X-ray luminosities the predicted dependence of $f_{\text {bursty }}$ on $L_{\mathrm{X}}$ is different for the obscured and unobscured AGN populations. Indeed, for the latter, $f_{\text {bursty }}$ is a monotonically increasing function of $L_{\mathrm{X}}$, while for obscured AGNs $f_{\text {bursty }}$ initially increases with $L_{X}$ (similarly to the unobscured population) and then decreases. This is due to the fact that obscured AGNs correspond to early stages of feedback action; in particular for a given orientation of the line of sight, the observed column density depends on the time elapsed since the start of the blast wave expansion. The faster expansion characterizing the blast wave of luminous AGNs thus corresponds to a larger probability that we will observe them as unobscured AGNs. Thus, the predicted fraction of obscured AGN decreases with increasing AGN luminosity (Menci et al. 2008). Moreover, the probability of finding a luminous AGN in a gas rich galaxy is low for galaxies with high values of SFR owing to the energy released into the interstellar medium by SNae feedback.

\subsection{The starburstiness of high-z QSOs}

The results shown in the previous sections indicate that the measurements of SFR and $M_{*}$ of luminous AGNs are fundamental to constrain AGN triggering mechanisms. Indeed, interactiondriven models for AGNs predict that a large fraction $(\gtrsim 0.8)$ of galaxies hosting high-luminosity AGNs $\left(L_{\mathrm{X}} \gtrsim 10^{44.5} \mathrm{erg} / \mathrm{s}\right)$ have SSFRs large enough to be selected as starburst $\left(R_{\mathrm{SB}}>4\right)$. This is valid at all epochs, as it is shown in Fig. 5 (left panel) where the predicted $f_{\text {bursty }}$ is given as a function of $z$ for three different AGN luminosity bins.

At $z \lesssim 0.5$ the evolution of $f_{\text {bursty }}$ is similar for AGNs with different luminosity. The increase of $f_{\text {bursty }}$ with $z$ is due to the decrease of the fraction of massive galaxies $\left(M_{*} \geq 10^{12} M_{\odot}\right)$ hosting AGNs with these luminosities (see Fig. 2). This similar evolution implies that the slope of the $f_{\text {bursty }}-L_{X}$ relation remains almost unchanged up to $z \simeq 0.5$.

At higher redshifts the $f_{\text {bursty }}-L_{X}$ relation steepens. In fact, the fraction of starbursting systems hosting AGN with $L_{X}>$ $10^{44.5} \mathrm{erg} / \mathrm{s}$ remains nearly constant with $z$, while the fraction of starburst galaxies hosting lower luminosity AGNs decreases with redshift. The latter trend is determined by the increase of the normalization of the galaxy main sequence with redshift. The predicted evolution of the galaxy main sequence, which determines the position of the peak at $z \simeq 0.5$ of the $f_{\text {bursty }}$ evolution for low-luminosity AGNs, is driven by the larger amount of cold gas available for star formation at earlier epochs and by the shorter star formation timescale $\tau_{q} \propto t_{\mathrm{d}}$ of high- $z$ galaxies. Despite the increase of the main sequence's normalization with redshift, the model predicts that galaxies hosting highluminosity AGNs lie well above the galaxy main sequence at all redshifts. To test this prediction we estimate the starburstiness of the high- $z$ QSOs with $L_{\mathrm{bol}} \geq 10^{46} \mathrm{erg} / \mathrm{s}\left(L_{\mathrm{X}} \gtrsim 10^{44.5} \mathrm{erg} / \mathrm{s}\right)$ belonging to the heterogeneous sample described in Sect. 3. To estimate $R_{\mathrm{SB}}$ in these objects we conservatively adopted SFRs derived by assuming that the IR luminosity is dominated by the FIR emission in these objects and considering the AGN contribution in the FIR (Sect. 3). To estimate the stellar masses of the CO-detected QSOs from the difference between dynamical 
A. Lamastra et al.: Probing AGN triggering mechanisms through the starburstiness of the host galaxies
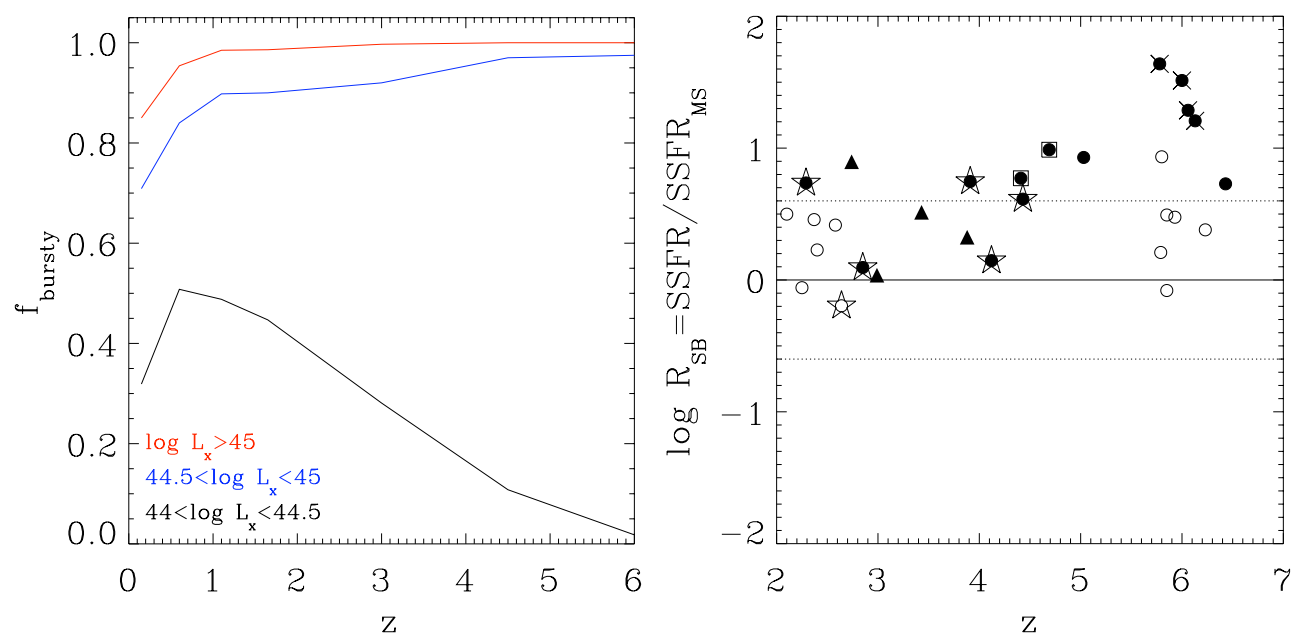

Fig. 5. Left: $f_{\text {bursty }}$ as a function of $z$ for three different AGN luminosity bins: $44<\log L_{X} / \mathrm{erg} \mathrm{s}^{-1}<44.5,44.5<\log L_{X} / \mathrm{erg} \mathrm{s}^{-1}<45$, and $\log L_{\mathrm{X}} / \mathrm{erg} \mathrm{s}^{-1}>45$ from bottom to top. Right: the starburstiness $R_{\mathrm{SB}}$ as a function of $z$ of QSOs with $L_{\mathrm{bol}}>10^{46} \mathrm{erg} / \mathrm{s}$. Triangles: stellar masses from SED fitting (Polletta et al. 2008; Lacy et al. 2011); circles: stellar masses from dynamical masses within the CO emitting region (Wang et al. 2010; Solomon \& Vanden Bout 2005; Coppin et al. 2008; Shields et al. 2006; Maiolino et al. 2007; Gallerani 2012) and the [CII] emitting region (crossed circles, Wang et al. 2013). For the sources with [CII] measurements $M_{\text {dyn }}$ are calculated assuming the disk inclination angle estimated from the [CII] minor and major axes ratio (see Wang et al. 2013). Open circles indicate dynamical masses derived assuming a disk radius of 2-2.5 kpc, while filled circles indicate dynamical masses obtained from spatially resolved measurements of the molecular gas emitting region. Squared circles denote sources in which $R$ is measured as half the component separation in merger model (see Shields et al. 2006). Starred circles denote gravitationally lensed QSOs for which the CO and FIR luminosities have been corrected for magnification (Riechers 2011).

and gas masses, for the latter we neglect the contribution of the atomic gas and we assume $\alpha_{\mathrm{CO}}=0.8\left(\mathrm{~K} \mathrm{~km} \mathrm{~s}^{-1} \mathrm{pc}^{2}\right)^{-1}$. This value is lower than the one used for disk galaxies $\left(\alpha_{\mathrm{CO}} \gtrsim\right.$ $4\left(\mathrm{~K} \mathrm{~km} \mathrm{~s}^{-1} \mathrm{pc}^{2}\right)^{-1}$, e.g. Daddi et al. 2010b; Genzel et al. 2010; Magdis et al. 2011; Magnelli et al. 2012). Although the exact value of $\alpha_{\mathrm{CO}}$ for starburst and disk galaxies is a debated topic (see Bolatto et al. 2013, for a review), the value of $\alpha_{\mathrm{CO}}$ assumed in this work corresponds to lower gas masses for a given $\mathrm{CO}$ line luminosity for QSO hosts than for disk galaxies. Thus our estimates of the stellar masses represent upper limits. We use the galaxy main sequences obtained by Santini et al. (2009), Daddi et al. (2009), and Stark et al. (2009) for the redshift intervals $2<z<2.5,2.5<z<4$, and $z>4$, respectively. The resulting $R_{\mathrm{SB}}$ is shown as function of $z$ in Fig. 5 (right panel). We find that $\sim 90 \%$ (26/29) of these QSOs lies above the galaxy main sequence and that $\sim 45 \%(13 / 29)$ has $R_{\mathrm{SB}} \geq 4$. Although the latter fraction is lower than that predicted by the model $(\gtrsim 0.8$, see left panel of 5), it represents a lower limit owing to the conservative SFR and $M_{*}$ estimates that we have adopted. This analysis suggests that on average the host of luminous QSO are more "starbursty" than normal star forming galaxies, bearing in mind the large uncertainties in the estimation of the stellar masses in these objects. A similar trend is also found for radio AGNs at $z<2$ (Karouzos et al. 2013).

\section{Conclusions}

We have investigated the star formation properties of the hosts of luminous $\left(L_{X} \geqslant 10^{44} \mathrm{erg} / \mathrm{s}\right)$ AGNs predicted under the assumption that starburst events and AGN activity are triggered by galaxy encounters during their merging histories. The latter are described through Monte Carlo realizations and are connected to star formation and $\mathrm{BH}$ accretion using a SAM of galaxy formation in a cosmological framework. We compared the model predictions with new measurements of the fraction of AGNs hosted in starburst galaxies as a function of the AGN luminosity in the redshift range $0.3<z<6.5$ to constrain AGN triggering mechanisms. The main results of this paper follow.

- Pinning down the AGN triggering mechanism from the relation between the SFR of the host galaxy and the AGN luminosity is a difficult task. On the observational side, the observed relation is still uncertain due to observational bias and instrumental limitation in sensitivity. On the theoretical side, even a model based on galaxy interactions as triggers of AGN and starburst activities, predicts a large scatter $(\simeq 3$ orders of magnitude) at low AGN luminosities owing to the large contribution of the quiescent component of star formation to the total SFR of the galaxy in these sources.

- The relation between the AGN luminosity and the fraction $f_{\text {bursty }}$ of AGNs hosted in starburst galaxies is a powerful tool to constrain AGN triggering mechanisms since the starburstiness $R_{\mathrm{SB}}=S S F R / S S F R_{\mathrm{MS}}$ of the host galaxy is an effective diagnostic to separate the quiescent and starburst modes of star formation (Lamastra et al. 2013). By adopting a starburst threshold of $R_{\mathrm{SB}}>4$ (Rodighiero et al. 2011; Sargent et al. 2012) we find that the predicted fraction $f_{\text {bursty }}$ increases with AGN X-ray luminosity from $\lesssim 0.2$ at $L_{\mathrm{X}} \lesssim 10^{44} \mathrm{erg} / \mathrm{s}$ to $\gtrsim 0.9$ at $L_{\mathrm{X}} \gtrsim 10^{45} \mathrm{erg} / \mathrm{s}$ over a wide redshift interval from $z \simeq 0$ to $z \simeq 6$.

- Interaction-driven models including AGN feedback related to the luminous AGN phase predict that at low X-ray luminosities $\left(L_{\mathrm{X}} \lesssim 10^{44} \mathrm{erg} / \mathrm{s}\right) f_{\text {bursty }}$ increases with $L_{\mathrm{X}}$ similarly for unobscured and obscured AGNs, while at higher luminosities $f_{\text {bursty }}$ steeply increases and decreases for the unobscured and obscured populations, respectively.

- The sharp, steep relation between $f_{\text {bursty }}$ and the AGN luminosity predicted by interaction-driven models implies that a large fraction $(\simeq 80 \%)$ of luminous AGNs $\left(L_{X} \geq 10^{44.5} \mathrm{erg} / \mathrm{s}\right)$ are in starburst galaxies. At present, observations indicate that at least $\simeq 50 \%$ of the QSO hosts at $2<z<6.5$ are starburst galaxies. Future systematic studies of the stellar properties of high luminosity AGNs are therefore necessary in 
order to make a step forward in our understanding of AGN triggering mechanisms.

Acknowledgements. The authors thank Benjamin Magnelli for kindly providing the SFR of HS1700+6416, and the referee for helpful comments. This work was supported by ASI/INAF contracts I/024/05/0 and I/009/10/0 and PRIN INAF 2011, 2013.

\section{References}

Alexander, D. M., Brandt, W. N., Smail, I., et al. 2008, AJ, 135, 1968 Barnes, J. E., \& Hernquist, L. E. 1991, ApJ, 370, L65

Barnes, J. E., \& Hernquist, L. 1996, ApJ, 471, 115

Bennert, N., Canalizo, G., Jungwiert, B., et al. 2008, ApJ, 677, 846

Bernardi, M., Meert, A., Sheth, R. K., et al. 2013, MNRAS, 436, 697

Blitz, L., \& Rosolowsky, E. 2006, ApJ, 650, 933

Bolatto, A. D., Wolfire, M., \& Leroy, A. K. 2013, ARA\&A, 51, 207

Bongiorno, A., Merloni, A., Brusa, M., et al. 2012, MNRAS, 427, 3103

Bournaud, F., Dekel, A., Teyssier, R., et al. 2011, ApJ, 741, L33

Bournaud, F., Juneau, S., Le Floc'h, E., et al. 2012, ApJ, 757, 81

Bower, R. G., Benson, A. J., Malbon, R., et al. 2006, MNRAS, 370, 645

Brinchmann, J., Charlot, S., White, S. D. M., et al. 2004, MNRAS, 351, 1151

Brusa, M., Civano, F., Comastri, A., et al. 2010, ApJ, 716, 348

Cappelluti, N., Brusa, M., Hasinger, G., et al. 2009, A\&A, 497, 635

Cavaliere, A., \& Vittorini, V. 2000, ApJ, 543, 599

Chary, R., \& Elbaz, D. 2001, ApJ, 556, 562

Chen, C.-T. J., Hickox, R. C., Alberts, S., et al. 2013, ApJ, 773, 3

Coppin, K. E. K., Swinbank, A. M., Neri, R., et al. 2008, MNRAS, 389, 45

Cox, T. J., Jonsson, P., Somerville, R. S., Primack, J. R., \& Dekel, A. 2008, MNRAS, 384, 386

Croton, D. J. 2006, MNRAS, 369, 1808

Croton, D. J., Springel, V., White, S. D. M., et al. 2006, MNRAS, 365, 11

Daddi, E., Dickinson, M., Morrison, G., et al. 2007, ApJ, 670, 156

Daddi, E., Dannerbauer, H., Stern, D., et al. 2009, ApJ, 694, 1517

Daddi, E., Bournaud, F., Walter, F., et al. 2010a, ApJ, 713, 686

Daddi, E., Elbaz, D., Walter, F., et al. 2010b, ApJ, 714, L118

Dale, D. A., \& Helou, G. 2002, ApJ, 576, 159

Damen, M., Labbé, I., Franx, M., et al. 2009, ApJ, 690, 937

Davé, R. 2008, MNRAS, 385, 147

Di Matteo, T., Springel, V., \& Hernquist, L. 2005, Nature, 433, 604

Elbaz, D., Daddi, E., Le Borgne, D., et al. 2007, A\&A, 468, 33

Elbaz, D., Dickinson, M., Hwang, H. S., et al. 2011, A\&A, 533, A119

Engel, H., Tacconi, L. J., Davies, R. I., et al. 2010, ApJ, 724, 233

Fanidakis, N., Baugh, C. M., Benson, A. J., et al. 2012, MNRAS, 419, 2797

Ferrarese, L., \& Merritt, D. 2000, ApJ, 539, L9

Fontanot, F., De Lucia, G., Monaco, P., Somerville, R. S., \& Santini, P. 2009, MNRAS, 397, 1776

Fu, H., Cooray, A., Feruglio, C., et al. 2013, Nature, 498, 338

Gallerani, S. 2012 [arXiv: 1211.0695]

Geach, J. E., Smail, I., Moran, S. M., et al. 2011, ApJ, 730, L19

Gebhardt, K., Bender, R., Bower, G., et al. 2000, ApJ, 539, L13

Genzel, R., Tacconi, L. J., Gracia-Carpio, J., et al. 2010, MNRAS, 407, 2091

González, V., Labbé, I., Bouwens, R. J., et al. 2011, ApJ, 735, L34

Guo, Q., White, S., Boylan-Kolchin, M., et al. 2011, MNRAS, 413, 101

Häring, N., \& Rix, H.-W. 2004, ApJ, 604, L89

Hernquist, L. 1989, Nature, 340, 687

Hickox, R. C., Mullaney, J. R., Alexander, D. M., et al. 2013, ApJ, submitted [arXiv: 1306.3218]

Hirschmann, M., Khochfar, S., Burkert, A., et al. 2010, MNRAS, 407, 1016

Hirschmann, M., Somerville, R. S., Naab, T., \& Burkert, A. 2012, MNRAS, 426, 237

Ho, L. 1999, in Observational Evidence for the Black Holes in the Universe, ed.

S. K. Chakrabarti, Astrophys. Space Sci. Lib., 234, 157

Hopkins, P. F., Hernquist, L., Cox, T. J., et al. 2006, ApJS, 163, 1

Jahnke, K., \& Macciò, A. V. 2011, ApJ, 734, 92

Karouzos, M., Im, M., Trichas, M., et al. 2013, ApJ, submitted [arXiv: 1309.7353]

Kauffmann, G., \& Haehnelt, M. 2000, MNRAS, 311, 576

Kennicutt, Jr., R. C. 1998, ApJ, 498, 541

Kim, D.-C., Veilleux, S., \& Sanders, D. B. 1998, ApJ, 508, 627

Kormendy, J., \& Bender, R. 2009, ApJ, 691, L142

Kormendy, J., \& Richstone, D. 1995, ARA\&A, 33, 581

Lacy, M., Petric, A. O., Martínez-Sansigre, A., et al. 2011, AJ, 142, 196
Lamastra, A., Menci, N., Maiolino, R., Fiore, F., \& Merloni, A. 2010, MNRAS, 405, 29

Lamastra, A., Menci, N., Fiore, F., \& Santini, P. 2013, A\&A, 552, A44

Lanzuisi, G., Giustini, M., Cappi, M., et al. 2012, A\&A, 544, A2

Lin, L., Dickinson, M., Jian, H.-Y., et al. 2012, ApJ, 756, 71

Lutz, D., Sturm, E., Tacconi, L. J., et al. 2008, ApJ, 684, 853

Lutz, D., Mainieri, V., Rafferty, D., et al. 2010, ApJ, 712, 1287

Lutz, D., Poglitsch, A., Altieri, B., et al. 2011, A\&A, 532, A90

Magdis, G. E., Daddi, E., Elbaz, D., et al. 2011, ApJ, 740, L15

Magdis, G. E., Daddi, E., Béthermin, M., et al. 2012, ApJ, 760, 6

Magnelli, B., Saintonge, A., Lutz, D., et al. 2012, A\&A, 548, A22

Magorrian, J., Tremaine, S., Richstone, D., et al. 1998, AJ, 115, 2285

Mainieri, V., Hasinger, G., Cappelluti, N., et al. 2007, ApJS, 172, 368

Mainieri, V., Bongiorno, A., Merloni, A., et al. 2011, A\&A, 535, A80

Maiolino, R., Neri, R., Beelen, A., et al. 2007, A\&A, 472, L33

Marconi, A., \& Hunt, L. K. 2003, ApJ, 589, L21

Marconi, A., Risaliti, G., Gilli, R., et al. 2004, MNRAS, 351, 169

Marulli, F., Bonoli, S., Branchini, E., Moscardini, L., \& Springel, V. 2008, MNRAS, 385, 1846

Menci, N., Cavaliere, A., Fontana, A., et al. 2003, ApJ, 587, L63

Menci, N., Cavaliere, A., Fontana, A., et al. 2004, ApJ, 604, 12

Menci, N., Fontana, A., Giallongo, E., \& Salimbeni, S. 2005, ApJ, 632, 49

Menci, N., Fontana, A., Giallongo, E., Grazian, A., \& Salimbeni, S. 2006, ApJ, 647, 753

Menci, N., Fiore, F., Puccetti, S., \& Cavaliere, A. 2008, ApJ, 686, 219

Mihos, J. C., \& Hernquist, L. 1994, ApJ, 431, L9

Mihos, J. C., \& Hernquist, L. 1996, ApJ, 464, 641

Mitchell, P. D., Lacey, C. G., Baugh, C. M., \& Cole, S. 2013, MNRAS, 435, 87

Mo, H. J., Mao, S., \& White, S. D. M. 1998, MNRAS, 295, 319

Monaco, P., Fontanot, F., \& Taffoni, G. 2007, MNRAS, 375, 1189

Mullaney, J. R., Daddi, E., Béthermin, M., et al. 2012a, ApJ, 753, L30

Mullaney, J. R., Pannella, M., Daddi, E., et al. 2012b, MNRAS, 419, 95

Narayan, C. A., \& Jog, C. J. 2002, A\&A, 394, 89

Neistein, E., \& Netzer, H. 2013, MNRAS, submitted [arXiv: 1302 . 1576]

Netzer, H. 2009, MNRAS, 399, 1907

Netzer, H., Lutz, D., Schweitzer, M., et al. 2007, ApJ, 666, 806

Noeske, K. G., Weiner, B. J., Faber, S. M., et al. 2007, ApJ, 660, L43

Obreschkow, D., \& Rawlings, S. 2009, MNRAS, 400, 665

Pannella, M., Carilli, C. L., Daddi, E., et al. 2009, ApJ, 698, L116

Peng, C. Y. 2007, ApJ, 671, 1098

Pilbratt, G. L., Riedinger, J. R., Passvogel, T., et al. 2010, A\&A, 518, L1

Poglitsch, A., Waelkens, C., Geis, N., et al. 2010, A\&A, 518, L2

Polletta, M., Omont, A., Berta, S., et al. 2008, A\&A, 492, 81

Polletta, M., Nesvadba, N. P. H., Neri, R., et al. 2011, A\&A, 533, A20

Riechers, D. A. 2011, ApJ, 730, 108

Rodighiero, G., Daddi, E., Baronchelli, I., et al. 2011, ApJ, 739, L40

Rosario, D. J., Santini, P., Lutz, D., et al. 2012, A\&A, 545, A45

Rovilos, E., Comastri, A., Gilli, R., et al. 2012, A\&A, 546, A58

Salim, S., Rich, R. M., Charlot, S., et al. 2007, ApJS, 173, 267

Sanders, D. B., \& Mirabel, I. F. 1996, ARA\&A, 34, 749

Santini, P., Fontana, A., Grazian, A., et al. 2009, A\&A, 504, 751

Santini, P., Fontana, A., Grazian, A., et al. 2012, A\&A, 538, A33

Sargent, M. T., Béthermin, M., Daddi, E., \& Elbaz, D. 2012, ApJ, 747, L31

Saslaw, W. C. 1985, Gravitational physics of stellar and galactic systems (Cambridge: Cambridge University Press)

Schweitzer, M., Lutz, D., Sturm, E., et al. 2006, ApJ, 649, 79

Scoville, N., Aussel, H., Brusa, M., et al. 2007, ApJS, 172, 1

Shao, L., Lutz, D., Nordon, R., et al. 2010, A\&A, 518, L26

Shields, G. A., Menezes, K. L., Massart, C. A., \& Vanden Bout, P. 2006, ApJ, 641,683

Solomon, P. M., \& Vanden Bout, P. A. 2005, ARA\&A, 43, 677

Soltan, A. 1982, MNRAS, 200, 115

Somerville, R. S., Hopkins, P. F., Cox, T. J., Robertson, B. E., \& Hernquist, L. 2008, MNRAS, 391, 481

Stark, D. P., Ellis, R. S., Bunker, A., et al. 2009, ApJ, 697, 1493

Tacconi, L. J., Genzel, R., Smail, I., et al. 2008, ApJ, 680, 246

Tacconi, L. J., Genzel, R., Neri, R., et al. 2010, Nature, 463, 781

Tran, Q. D., Lutz, D., Genzel, R., et al. 2001, ApJ, 552, 527

Treister, E., Schawinski, K., Urry, C. M., \& Simmons, B. D. 2012, ApJ, 758, L39

Veilleux, S., Kim, D.-C., \& Sanders, D. B. 1999, ApJ, 522, 113

Wang, R., Carilli, C. L., Neri, R., et al. 2010, ApJ, 714, 699

Wang, R., Wagg, J., Carilli, C. L., et al. 2013, ApJ, 773, 44

Weinmann, S. M., Neistein, E., \& Dekel, A. 2011, MNRAS, 417, 2737

Whitaker, K. E., van Dokkum, P. G., Brammer, G., \& Franx, M. 2012, ApJ, 754, L29 